\title{
A importância da saúde bucal na reabilitação psicossocial: Sorrir e cuidar em saúde mental
}

\author{
The importance of oral health in psychosocial rehabilitation: Smiling and caring in mental health
}

La importancia de la salud bucal en la rehabilitación psicosocial: Sonreír y cuidar en salud mental

Recebido: 01/04/2021 | Revisado: 06/04/2021 | Aceito: 13/04/2021 | Publicado: 25/04/2021

Rosane Mara Pontes de Oliveira

ORCID: https://orcid.org/0000-0002-2183-1423

Escola de Enfermagem Anna Nery, Brasil

E-mail: romapope@gmail.com

Nilton Gonçalves de Oliveira Junior

ORCID: https://orcid.org/0000-0002-6356-2292

Associação Brasileira de Odontologia, Brasil

E-mail: drnilton@gmail.com

Paula Cristina da Silva Cavalcanti

ORCID: https://orcid.org/0000-0001-7424-2043

Associação Educacional Dom Bosco, Brasil

E-mail: paulacristinadasilva@yahoo.com.br

Manoela Alves

ORCID: https://orcid.org/0000-0003-4239-9577

Universidade de Vassouras, Brasil

E-mail: alves.manoela@gmail.com

Maria Giovana Borges Saidel

ORCID: https://orcid.org/0000-0002-3259-1760

Faculdade de Enfermagem, Brasil

E-mail: mgsaidel@unicamp.br

Virginia Faria Damasio Dutra

ORCID: https://orcid.org/0000-0001-5531-9623

Escola de Enfermagem Anna Nery, Brasil

E-mail: virginia.damasio@gmail.com

Cristina Maria Douat Loyola

ORCID: https://orcid.org/0000-0003-2824-6531

Universidade Ceuma, Brasil

E-mail: crisloyola@hotmail.com

\begin{abstract}
Resumo
A saúde bucal faz parte da saúde física e mental, pois interfere tanto na mastigação e digestão quanto na possibilidade de comunicação. O objetivo deste estudo foi identificar as condições de saúde bucal de pacientes internados em uma instituição psiquiátrica e discutir implicações para os processos de reabilitação psicossocial. O estudo foi realizado com 104 pacientes, utilizando o odontograma e entrevista semiestruturada individual para a coleta de dados. Os dados quantitativos foram analisados utilizando-se a estatística descritiva e o índice CPO-D, apresentando resultados por frequências absolutas e relativas e medidas de tendência central, os dados qualitativos foram tratados por meio da técnica de análise de conteúdo. Os resultados evidenciaram índice de CPO-D médio de 19,41 para homens e 20,38 para mulheres e perda total de dentes nas mulheres de $29 \%$ e nos homens de 6,06\%. Da análise do conteúdo emergiu duas categorias: "Ausência total de dentes (edêntulas) interferindo na comunicação" e "Ausência de dentes e a autoimagem/estética". Conclui-se que a relação entre a saúde bucal e estratégias de reabilitação psicossocial precisam ser consideradas no decorrer do processo terapêutico pela equipe multiprofissional.
\end{abstract}

Palavras-chave: Saúde mental; Saúde bucal; Unidade hospitalar de psiquiatria; Sistemas de apoio psicossocial; Impacto psicossocial.

\footnotetext{
Abstract

Oral health is part of physical and mental health, as it interferes with both chewing and digestion and the possibility of communication. The aim of this study was to identify the oral health conditions of patients admitted to a psychiatric institution and to discuss implications for psychosocial rehabilitation processes. The study was carried out with 104 patients, using the odontogram and individual semi-structured interview for data collection. Quantitative data were analyzed using descriptive statistics and the DMFT index. The results included absolute and relative frequencies and measures of central tendency. Qualitative data were treated using the content analysis technique. The results showed an average DMFT index of 19.41 for men and 20.38 for women and total loss of teeth of $29 \%$ in women and $6.06 \%$ in
} 
men. Two categories emerged from the content analysis: "Total absence of teeth (edentulous) interfering with communication" and "Absence of teeth and self-image/aesthetics". It is concluded that the relationship between oral health and psychosocial rehabilitation strategies needs to be considered during the therapeutic process by the multidisciplinary team.

Keywords: Mental health; Oral health; Hospital psychiatric department; Psychosocial support systems; Psychosocial impact.

\section{Resumen}

La salud bucal es parte de la salud física y mental, ya que interfiere tanto con la masticación como con la digestión y la posibilidad de comunicación. El objetivo de este estudio fue identificar las condiciones de salud bucal de los pacientes ingresados en una institución psiquiátrica y discutir las implicaciones para los procesos de rehabilitación psicosocial. El estudio se realizó con 104 pacientes, utilizando el odontograma y la entrevista semiestructurada individual para la recolección de datos. Los datos cuantitativos se analizaron mediante estadística descriptiva y el índice CPOD. Los resultados incluyeron frecuencias absolutas y relativas y medidas de tendencia central. Los datos cualitativos se trataron mediante la técnica de análisis de contenido. Los resultados mostraron un índice CPOD promedio de 19,41 para los hombres y 20,38 para las mujeres y una pérdida total de dientes del $29 \%$ en las mujeres y del 6,06\% en los hombres. Del análisis de contenido surgieron dos categorías: "Ausencia total de dientes (desdentados) que interfieren con la comunicación" y "Ausencia de dientes y autoimagen / estética". Se concluye que la relación entre la salud bucal y las estrategias de rehabilitación psicosocial debe ser considerada durante el proceso terapéutico por parte del equipo multidisciplinario.

Palabras clave: Salud mental; Salud bucal; Servicio de psiquiatría en hospital; Sistemas de apoyo psicosocial; Impacto psicosocial.

\section{Introdução}

A saúde bucal faz parte da saúde física e mental, porque interfere tanto na mastigação e digestão de alimentos, quanto na possibilidade de comunicação, de passar e receber mensagens não verbais, além de influenciar diretamente a estética perceptível ao primeiro olhar. A saúde oral está relacionada à saúde sistêmica, doenças do coração, pulmão, próstata, osteoporose, diabetes e até câncer podem ser evitados com simples cuidados com a higiene e saúde dos dentes (Beaudette et al., 2017).

A perda dentária é considerada um dos principais agravos à saúde bucal devido à sua alta prevalência, aos danos estéticos, funcionais, psicológicos e sociais que acarreta. A ausência de dentes total ou parcial (edentulismo) interfere na autoimagem e na aceitação do indivíduo pela sociedade. Essas perdas ocorrem como consequência de eventos genéticos ou adquiridos (mutilatórios), que se sucedem ao longo da vida. Mesmo com avanços obtidos nos últimos anos na prevenção e tratamento de doenças que comprometem a dentição adulta, ainda é comum no Brasil a perda dos dentes e consequente, edentulismo (Gomes Filho et al., 2019). Ao cuidar da saúde bucal, atuamos sobre a saúdes física, psíquica e social do cidadão. A articulação destas esferas da vida aponta para a qualidade de vida das pessoas, que somam à dificuldade de socialização, por vezes decorrentes do transtorno mental, a vergonha ou inibição adicional de conversar, sorrir ou até mesmo de conseguir um emprego (U.S. Department of Health and Human Services, 2016). A ideia do estudo centra-se na saúde bucal dos pacientes internados em clínicas psiquiátricas, como uma estratégia de diminuir o estigma, aumentar o nível de saúde e facilitar a reinserção social.

A prática tem demonstrado que pacientes com internação de longa permanência que perdem o contato social apresentam aspectos de desvalorização do autocuidado. Somado a isso, a utilização rotineira de psicofármacos provoca redução de fluxo e volume salivar, fator fundamental na manutenção da homeostasia do meio bucal (Falcão et al., 2013), além do comprometimento da coordenação motora, dificultando sobremaneira a higiene oral dos indivíduos com transtornos mentais (Hill et al., 2016). Portanto a saúde bucal, independente do modelo assistencial psiquiátrico ao qual estão submetidos, deve ser avaliada criteriosamente, haja vista, que a condição bucal é uma variável clínica que interfere na saúde mental (Beaudette et al., 2017). 
É evidente, pelas afirmações acima que há a necessidade de uma avaliação pautada nos conceitos de clínica ampliada, considerando os princípios de humanização e integralidade das políticas de saúde mental. O movimento da Reforma Psiquiátrica com a reconfiguração da assistência ao indivíduo com transtorno mental, propõe uma política de cuidado centrada no sujeito, com práticas reabilitadoras que facilitem a inclusão na vida em sociedade, além de oferecer equipamentos substitutivos às internações de longa permanência em instituições de caráter asilar (Esperidão et al., 2013).

Essas questões estão em consonância com as "Ações de Reabilitação bucal" da Política Nacional de Saúde Bucal do Ministério da Saúde que consistem na recuperação parcial ou total das capacidades perdidas como resultado da doença e na reintegração do indivíduo ao seu ambiente social e a sua atividade profissional (Ministério da Saúde, 2004). A reabilitação psicossocial aponta para prover o ótimo nível de funcionamento de indivíduos e sociedade e a minimização de incapacidades e desvantagem física ou mental, acentuando escolhas individuais em como viver prosperamente na comunidade (Petersen et al., 2005).

Tem-se o consenso universal de que os serviços de reabilitação psiquiátrica são destinados a ajudar pessoas com doença mental severa para que estes alcancem recuperação; atinjam máxima integração comunitária e alta possibilidade de qualidade de vida (Almeida et al., 2010; Esperidão et al., 2013). Trata-se de uma prática de articulação, de preservação ou de resgate da história de vida das pessoas. Mostra, também, que, enquanto muitos profissionais "esperam" eternamente as condições ideais de vida, que às vezes não chegam nunca, outros, criativos e envolvidos, faz de cada lugar, de cada momento, a oportunidade para pôr em prática a reabilitação, no cotidiano real da vida das pessoas (Sena et al., 2019).

Considerando esta exposição, diversos questionamentos buscaram refletir sobre quais as condições de saúde bucal dos pacientes internados em instituições psiquiátricas e suas implicações para o processo de reabilitação social. Essas reflexões por meio da pesquisa de campo objetivaram identificar as condições de saúde bucal de pacientes internados em instituição psiquiátrica e discutir implicações para o processo de reabilitação psicossocial. Identificar as condições de saúde bucal de pacientes internados em uma instituição psiquiátrica e discutir implicações para os processos de reabilitação psicossocial

\section{Metodologia}

Trata-se de um estudo observacional, de caráter transversal (Polit; Beck \& Hungler, 2011) com pacientes internados em uma clínica psiquiátrica conveniada ao Sistema Único de Saúde (SUS) da região centro sul fluminense no Estado do Rio de Janeiro, Brasil. O campo da pesquisa foi um Hospital Psiquiátrico que atende pacientes da região Centro Sul Fluminense. Possui dois grandes setores: enfermaria masculina e enfermaria feminina, com 80 e 40 leitos, respectivamente. Sobre os aspectos éticos, foram seguidas as exigências éticas estabelecidas pela Resolução no 466/2012, do Conselho Nacional de Saúde, o projeto foi apresentado à instituição e encaminhado ao CEP da Escola de Enfermagem Anna Nery/UFRJ e aprovado sob parecer de $\mathrm{n}^{\circ} .856 .583$ em 04 de novembro de 2014. Os participantes e/ou os responsáveis assinaram o Termo de Consentimento Livre e Esclarecido e os dados foram protegidos por meio de códigos alfanuméricos.

Os critérios de inclusão foram: indivíduos que estavam internados no hospital que serviu de campo para este estudo (sem limites de tempo, todos os pacientes e/ou responsáveis que assinaram o TCLE foram incluídos no estudo); ambos os sexos e maiores de 18 anos. O critério de exclusão foi alguma condição clínica e/ou psíquica aguda no momento da coleta dos dados que impedia o exame físico oral e/ou a entrevista semiestruturada. Os dados foram coletados ao longo de 2014 e 2015 em três etapas (não havendo intervalo delimitado para as coletas).

Na primeira etapa foi realizado um exame clínico bucal detalhado por um odontólogo (componente da equipe de pesquisa e com experiência na psiquiatria). A segunda etapa foi o preenchimento do odontograma (também realizado pelo odontólogo) e, na terceira fase, foi realizada uma entrevista semiestruturada individual com os pacientes (conduzida por 
enfermeiras especialistas e com experiência em saúde mental). O roteiro da entrevista versou sobre as percepções dele em relação à saúde bucal e o processo de reabilitação psicossocial.

Para a análise dos dados quantitativos utilizou-se a estatística descritiva com auxílio do programa Excel Office, por meio do índice CPO-D (Cypriano; Sousa \& Wada, 2005; Petersen et al., 2005), cujo valor expressa a média do número de dentes cariados, perdidos e obturados. Quanto aos dados qualitativos, estes foram analisados por meio da técnica de análise conteúdo em três etapas, a pré-análise; exploração do material e tratamento dos resultados obtidos e interpretação (Bardin, 2011). Os resultados após análise quantitativa e qualitativa foram interpretados fundamentado a discussão à luz de referenciais teóricos de reabilitação psicossocial e enfermagem em saúde mental (Saraceno, 2011; Esperidião et al., 2013; Oliveira et al., 2016).

\section{Resultados}

A amostra final, foi de 104 pacientes, sendo que 66 eram do sexo masculino e 38 do sexo feminino. Os dados qualitativos estão descritos e evidenciados na Tabela 1. Os resultados obtidos evidenciaram um índice CPO-D médio de 19,41 para os homens e 20,38 para as mulheres, sendo considerado um índice elevado, especialmente em decorrência do grande número de dentes extraídos. Com relação aos dentes perdidos esses traduzem perda em várias áreas e várias dimensões, a saber: ausência em área anterior e ausência em área posterior.

Os dados qualitativos fizeram emergir duas categorias que tratara da ausência total de dentes interferindo na comunicação e a ausência de dentes e a autoimagem/estética. Esses dados subsidiaram todo o processo de discussão fundamentado nos referenciais já descrito no método do presente estudo.

Tabela 1 - Média do índice CPO-D e dos seus componentes, segundo o sexo, dos pacientes internados. Ausência total de dentes interferindo na comunic ação.

\begin{tabular}{|c|c|c|c|c|c|c|c|c|c|c|c|}
\hline \multirow[b]{2}{*}{ Sexo } & & \multicolumn{3}{|c|}{ Cariados } & \multicolumn{3}{|c|}{ Perdidos } & \multicolumn{3}{|c|}{ Obturados } & \multirow[t]{2}{*}{ CPO-D } \\
\hline & & $\mathrm{N}$ & $\%$ & Média & $\mathrm{N}$ & $\%$ & Média & $\mathrm{N}$ & $\%$ & Média & \\
\hline Masculino & 66 & 1021 & 62,9 & 15,46 & 31 & 45,6 & 0,47 & 230 & 63,0 & 3,48 & 19,41 \\
\hline Feminino & 38 & 603 & 37,1 & 15,86 & 37 & 54,4 & 0,97 & 135 & 37 & 3,55 & 20,38 \\
\hline
\end{tabular}

Fonte: Autores.

\subsection{Categoria 1. Ausência total de dentes (edêntulas) interferindo na comunicação}

A frequência de perda total de dentes nas mulheres foi maior (29\%) do que no sexo masculino $(6,06 \%)$. Há prejuízos na capacidade mastigatória, restrições alimentares, além dos impactos nos aspectos sociais e psicológicos. Sendo assim, alguns resultados foram captados apenas por meio da entrevista semiestruturada, conforme fala abaixo.

Eu não gosto de falar muito porque não tenho dente. (Entrevista. A.L.)

Esta fala nos remete ao fato que o edentulismo interfere dramaticamente na comunicação dos pacientes, o que contribui para o isolamento e a não participação no processo reabilitador. A perda dos dentes anteriores e posteriores afeta tanto a função mastigatória mais estrita, de triturar alimentos duros, como a capacidade de sentir prazer pelo sabor do alimento, que precisa circular dentro da boca para estimular a percepção dos sabores. Sabemos todos que o tempo gasto pelos pacientes 
institucionalizados, para as refeições, não ultrapassa 20 minutos, contradizendo a função socializante que as reuniões com alimentação possuem em nossa sociedade. A dieta, nestes locais costuma pastosa ou sem pastosa para atender a realidade dos pacientes.

A falta total de dentes produz uma inibição ou constrangimento pela maior possibilidade de perdigotos durante a fala, pela dificuldade na pronúncia de algumas consoantes, contribuindo, deste modo, para a falta de comunicação. Havendo dificuldades na comunicação, estaremos diante de um quadro de prejuízo, que pode acometer os indivíduos de formas variáveis.

\subsection{Categoria 2. Ausência de dentes e a autoimagem/estética}

Os dados dessa categoria apontam a cárie como a maior responsável pelo edentulismo parcial, no sexo masculino corresponde a um índice CPO-D muito alto, dos 66 homens obtivemos 1021 dentes cariados o que equivale a uma média de 15,86 e para as 38 mulheres 603 dentes cariados e uma média de 15,46.

A doença psiquiátrica tem uma clínica muito própria, com os tremores, com a condição motora afetada, a falta de compreensão e condições psíquicas para desenvolver atividades de vida cotidiana que podem dificultar a higienização, e que representam um fator de risco para desenvolvimento de cáries e doenças periodontais. Para além das questões clínicas que acometem os pacientes, tornando-os mais suscetíveis à doença bucal, há as questões próprias da instituição psiquiátrica, que negligencia a higiene oral dos pacientes, bem como a postura dos familiares que também não são atentos a este cuidado. Como na fala:

\section{Eu não escovo o dente porque não tenho escova e nem pasta. (Entrevista J.S.)}

A cárie e a doença periodontal foram às causas mais importantes da perda dentária, provocando impacto na autoestima desses indivíduos e prejudicando o prognóstico do tratamento da doença mental. A falta de dentes leva a um sentimento de constrangimento e isolamento social, em um círculo que se retroalimenta. Eles deixam de sorrir ou sorriem com a mão na boca, falam pouco e se acham feios, e tudo isso produz mais isolamento social, diminuição da autoestima e da possibilidade de sentir prazer na vida. Expresso na confissão:

\section{Eu não gosto de tirar foto porque não tenho dente, fico feio na foto. (Entrevista M.S.)}

A autoimagem expressa a percepção que a pessoa tem de si, sendo definida em termos de uma constelação de pensamentos, sentimentos e ações acerca do relacionamento do indivíduo com outras pessoas.

\section{Discussão}

Conforme resultados descritos o índice CPO-D médio foi considerado elevado, sendo importante enfatizar que essa média decorre especialmente do grande número de dentes extraídos, contudo não foi observado uso de prótese.

Existem pesquisas que indicam uma experiência média de cárie semelhante nos indivíduos com transtornos psíquicos e na população em geral (Braun et al., 2018; Rocha et al., 2019). Contudo, a proporção maior de dentes não tratados e perdidos sugere que a exodontia é o tratamento adotado com mais frequência (Santos et al., 2018). Em outro hospital psiquiátrico de grande porte, entre os 1.108 indivíduos com esquizofrenia avaliados, a idade foi a única variável independentemente associada e obteve um CPO-D > 8. A menor escolaridade e a maior permanência no hospital estavam relacionadas com cárie e número 
de dentes remanescentes (Chu et al., 2010). Lewis, Jagger e Treasure (2001), descreveu sobre o estado de saúde bucal de pacientes psiquiátricos internados e obteve a pontuação média CPO-D de 19,1. Essas duas pesquisas corroboram com os resultados do presente estudo, e ainda acrescentam que a higiene bucal da população era pobre, havia doença periodontal e necessidade de raspagem e polimento (Lewis, Jagger e Treasure, 2001; Chu et al., 2010). Essa é uma realidade comum em pacientes psiquiátricos que passaram por internações longas, essas afirmativas tanto podem ser experenciadas da prática cotidiana dos profissionais, quanto em estudos sobre a temática (Lewis et al, 2001; Rocha et al., 2019). As afirmativas de estudos destacados nesse artigo e os resultados do presente estudo, demonstram a importância de considerar esse aspecto da saúde como componente para a reabilitação psicossocial.

Os dados referentes a saúde bucal estão conectados com a questão global da vida e sugerem um abandono de si opondo-se ao cuidado de si. O cuidado de si é compreendido como: um conjunto de práticas de cuidados, objetivando afirmar a própria liberdade e subjetividades do sujeito; forma de cuidado de si voltada para uma condição ética/política de existência na esfera pública; e ainda os enfrentamentos contemporâneos das práticas de cuidado no Brasil a partir da Reforma Sanitária (Castro; Furegato \& Santos, 2010; Dutra \& Rocha, 2011).

As pessoas com histórico de transtorno mental apresentam um elevado risco de desenvolver desordens orais e ainda, pouco visitam um profissional da odontologia, procuram tardiamente por tratamento e encontram profissionais despreparados que os conduzem a soluções muitas vezes mutiladoras (Sacchetto et al., 2013; Santos et al., 2018). Essa afirmativa reforça os resultados encontrados, o n de dentes cariados na amostra soma 1624, e 365 já com sinais de obturação no exame clínico. Em relação as soluções mutiladoras, a amostra também demonstra consonância, pois 68 dentes foram perdidos.

A precária saúde oral dos pacientes em instituições psiquiátricas parece ser resultante de diversos fatores, dos quais, destacam-se: hábitos de higiene, condições socioeconômicas, autocuidado, condições institucionais, difícil acesso à tratamentos odontológicos preventivos e conservadores (Chu et al., 2010; Braun et al., 2018). Se esses fatores contribuem para os problemas odontológicos, soma-se a esses fatores as condições inerentes de pacientes psiquiátricos institucionalizados. O histórico de abandono e/ou perda de contato com os familiares, dificuldades de reinserção social e limitações cognitivas que impedem algumas ações autônomas (Saraceno, 2011).

Reflete, de fato, a desistência de si, do cuidado de si enquanto ser autônomo: dono do seu corpo, da sua história e elemento ativo do corpo social. A questão reabilitação psicossocial certamente perpassa a reabilitação física, oral e biológica. Ou seja, investir no cuidado de si, desde ajudar o sujeito na gerência de si, responsabilizar instituições que objetivam cuidar, até as questões de cidadania. No caso de edentulismo faz-se urgente a reabilitação oral através de prótese, com objetivo de devolver função à cavidade oral, tanto na mastigação, como na fala, respiração e recomposição dos dentes perdidos e estética contribuindo para o equilíbrio do sistema mastigatório e sociabilização do indivíduo (Ministério da Saúde, 2004). Além de proporcionar espaços de cuidados para este fim, bem como profissionais capacitados para o cuidado efetivo desta clientela (Chu et al., 2010; Rocha et al., 2019).

A falta de dentes, mesmo parcial, interfere na qualidade de vida das pessoas internadas porque os sentimentos de constrangimento, de sentir-se feio influenciam nas suas relações sociais e nos seus vínculos afetivos, o que é agravado pelas condições psíquicas desses indivíduos. Essas situações fazem com que os pacientes se excluam, diminuindo sua rede social e levando-os ao isolamento, fortalecendo o círculo sofrimento-exclusão-mais-sofrimento e dificultando o processo de reabilitação psicossocial.

Se considerarmos a reabilitação psicossocial como forma de aumentar as habilidades para vida (Almeida; Dimenstein \& Severo, 2010), o cuidado de si, para a autonomia, a cidadania e as redes pessoais, que compreendem um aparato para construção da identidade e consequentemente a restruturação do ser social, temos que repensar o cuidado odontológico dos pacientes psiquiátricos. A importância deste cuidado é ainda mais visível quando se objetiva a desinstitucionalização 
(Saraceno, 2011; Seabra et al., 2018).

Seja no cuidado para a desinstitucionalização ou nas etapas vivenciadas pelo interno, o processo perpassa as trocas subjetivas (autoestima, comunicação e identidade), aumento do poder contratual (comunicação, sedução e imagem) e ainda trocas de mercadorias e valores. A ideia de cuidado para reabilitação reporta à integralidade do ser biológico e, sobretudo, ao investimento no ser social que ele precisa ser para poder fazer trocas mais favoráveis que aquelas do hospital. O caminho inverso refere-se a experiências da identidade social institucionalizada, que já não interroga mais nem a si mesma nem ao mundo, mas limita-se a sobrepor-se às outras instituições existentes como um elemento a mais no panorama geral (Flores, 2020; Oliveira et al., 2016).

No entanto, a reabilitação psicossocial inclui sorrisos; que refletem identidades, trocas e possibilidades de cuidados. A boca, os dentes e o sorriso representam ao mesmo tempo a singularidade do sujeito e a ferramenta de comunicação em um processo de reabilitação. Investir na saúde bucal significa um passo importante na autoimagem, na liberdade de ser, na busca da cidadania e ao acesso às tecnologias de cuidado (Oliveira et al., 2016). Então pergunta: como avançar no acolhimento quando há constrangimento? Como investir no diálogo quando é difícil a compreensão? Como aumentar a autonomia sem investir no sujeito social ou dar voz? As respostas dessas perguntas devem nos fazer refletir como os resultados desse estudo podem contribuir com a prática clínica do cuidado da saúde bucal em unidades de internação psiquiátrica.

Dentre as possibilidades aponta-se as tecnologias do cuidado, nas quais, o cuidado passa a assumir uma centralidade, na medida em que se objetiva ações em saúde de maneira integral (Dutra \& Rocha, 2011). Ainda na tentativa de responder as questões emergidas na discussão, na análise da saúde oral dos participantes da pesquisa não aparecem próteses de qualquer natureza (visando a reabilitação oral), o que pode ser explicado em certa medida pela dificuldade de adesão às medidas terapêuticas em decorrência do transtorno mental. Saraceno (2011) escreve que o que se espera é a adoção de uma abordagem global da doença e da saúde, incorporando intervenções mais articuladas, interdisciplinares e inovadores, permitindo a construção de contextos de identidades diversas, composta de minúsculos determinantes de qualidade de vida. Ou seja, a cidadania como um direito em si: soma de direitos e identidades, positivos e negativos, com potência de troca social (Saraceno, 2011).

Uma limitação deste estudo foi discutir a reabilitação psicossocial de dentro de uma instituição total. A variável saúde bucal afetou aspectos importantes da reabilitação e o estudo reforçou a necessidade de cuidados da saúde bucal para o processo de reabilitação Psicossocial, resultados evidenciados nas categorias de análise dos dados qualitativos. Visar a reabilitação significa investir em todos os aspectos da vida, nem sempre ligados diretamente ao diagnóstico clínico, mas também orientados pela capacidade de comunicação, de relacionamento interpessoal, de ter uma identidade que faça troca social, que melhore a autoestima e a autoimagem, para poder "caber na vida", de alguma forma. Na vida cotidiana dos seres humanos, em geral, a autoestima reside, em grande parte, no reconhecimento, na avaliação e na nomeação, pelo outro, de como somos, ou de como nos apresentamos, de fato, pelos elogios que recebemos.

\section{Conclusão}

O estudo conclui que foi possível identificar as condições de saúde bucal de pacientes internados em instituições psiquiátricas na amostra estudada por meio dos instrumentos de coleta de dados. Ao confrontar os achados do estudo à luz dos referenciais teóricos propostos foi possível concluir a articulação da saúde bucal e os processos de vida dos indivíduos com transtornos mentais em regime de internação. Os achados demonstraram que o cuidado com os dentes é um aspecto relevante para ações que podem potencializar ações que objetivam a reabilitação social do sujeito, não devendo, portanto, ser negligenciado pelos atores sociais que participam ativamente do cuidado desse indivíduo: familiares, instituição e equipe de 
saúde.

E finalmente conclui-se que os resultados dessa investigação são potentes no sentido de fornecer informações de uma amostra que demonstram processos deficientes no cuidado da saúde bucal das pessoas com transtornos mentais em regime de internação, além de apontar que as consequências disso pode impactar na adesão das ações de reabilitação psicossocial. Essa afirmativa foi encontrada nos resultados qualitativos, contudo acredita-se que mais investigações qualitativas devem ocorrer, inclusive com outros atores sociais do contexto estudado.

Nas implicações para a prática clínica, os resultados oferecem indicações que podem subsidiar propostas futuras de manejo em unidades de internação. Esse manejo teria como objetivo transmutar a realidade vivenciada por essas pessoas, ou seja, superar o paradigma que influencia na extração dentária e mutiladora como única opção de tratamento bucal. Essa construção se faz por meio do fortalecimento dos profissionais nas equipes interdisciplinares, nas quais por meio da edificação do Projeto Terapêutico Singular seja possível ações de avaliação precoce e atividades de prevenção e promoção da saúde bucal que atenda o perfil e necessidade dessa população.

\section{Referências}

Almeida, K. S., Dimenstein, M. \& Severo, A. K. (2010). Empowerment and psychosocial care: notes on a mental health association. Interface - Comunicaçao, Saude, Educaçao, 14(34), 577-589. Http://www.scielosp.org/pdf/icse/v14n34/aop1410.pdf

Bardin, L.(2011). Análise de conteúdo. Edições 70.

Beaudette, J. R., Fritz, P. C., Sullivan, P. J., \& Ward, W. E. (2017). Oral Health, Nutritional Choices, and Dental Fear and Anxiety. Dentistry journal, 5(1), 8. https://doi.org/10.3390/dj5010008

Braun, P. C., Vieira, R. A., Cristiano, D. P., \& Sonego, F. G. F. (2018). Impacto da saúde bucal na qualidade de vida dos pacientes usuários do centro de atenção psicossocial II do Município de Criciúma/SC. Revista de Odontologia da Universidade de São Paulo, 30(2), 132-143. http://publicacoes.unicid.edu.br/index.php/revistadaodontologia/article/view/677

Castro, A. S., Furegato, A. R. F. \& Santos, J. L. (2010). Sociodemographic and Clinical Characteristics of Psychiatric Re-hospitalizations. Revista LatinoAmericana de Enfermagem, 18(4), 800-808. https://doi.org/10.1590/S0104-11692010000400020

Chu, K.-Y., Yang, N.-P., Chou, P., Chiu, H.-J. \& Chi, L.-Y. (2010). Factors associated with dental caries among institutionalized residents with schizophrenia in Taiwan: a cross-sectional study. BMC Public Health, 10, 482. https://doi.org/10.1186/1471-2458-10-482

Cypriano, S., Sousa, M. L. \& Wada, R. S. (2005). Evaluation of simplified DMFT indices in epidemiological surveys of dental caries. Revista de Saúde Pública, 39(2), 285-292. https://doi.org/10.1590/S0034-89102005000200021

Dutra, V. F. D. \& Rocha, R. M. (2011). O processo de desinstitucionalização psiquiátrica: subsídios para o cuidado integral. Revista Enfermagem UERJ, 19(3), 386-391. http://www.facenf.uerj.br/v19n3/v19n3a08.pdf

Esperidião, E., Silva, N. S., Caixeta, C. C. \& Rodrigues, J. (2013). The Psychiatric Nursing, ABEn and the Scientific Department of Psychiatric and Mental Health Nursing: progress and challenges. Revista brasileira de Enfermagem, 66, 171-176. https://doi.org/10.1590/S0034-71672013000700022

Falcão, D. P., Mota, L. M. H., Pires, A. L. \& Bezerra, A. C. B. (2013). Sialometry: aspects of clinical interest. Revista Brasileira de Reumatologia, 53(6), 525531. https://doi.org/10.1016/j.rbr.2013.03.001

Flores D. (2020). A importância dos profissionais de saúde na desinstitucionalização do estigma dos sujeitos com transtornos mentais. Revista Portuguesa de Enfermagem de Saúde Mental, 23(41). http://dx.doi.org/10.19131/rpesm.0271

Gomes Filho, V. V., Gondinho, B. V. C., Silva Junior, M. F., Cavalcante, D. F. B., Bulgareli, J. V., Sousa, M. L. R., Frias, A. C., Batista, M. J. \& Pereira, A. C. (2019). Tooth loss in adults: factors associated with the position and number of lost teeth. Revista de Saúde Pública, 53(9). https://doi.org/10.11606/s15188787.2019053001318

Hill, L. J., Mushtaq, F., O'Neill, L., Flatters, I., Williams, J. H., \& Mon-Williams, M. (2016). The relationship between manual coordination and mental health. European child \& adolescent psychiatry, 25(3), 283-295. https://doi.org/10.1007/s00787-015-0732-2

Lewis, S., Jagger, R. G. \& Treasure, E. (2001). The oral health of psychiatric in-patients in South Wales. Special care in dentistry, 21(5), 182-186. https://doi.org/10.1111/j.1754-4505.2001.tb00252.x

Ministério da Saúde (BR). (2004). Política Nacional de Saúde Bucal: Brasil Sorridente.

Oliveira, R. M. P., Alves, M., Porto, I. S. \& Cavalcanti, P. C. S. (2016). A clínica de enfermagem psiquiátrica e suas novas tecnologias de cuidado. Journal of Research: Fundamental Care Online, 8(1), 3922-3934. https://pesquisa.bvsalud.org/gim/resource/en/lil-776204?lang=en 
Research, Society and Development, v. 10, n. 5, e0610514578, 2021

(CC BY 4.0) | ISSN 2525-3409 | DOI: http://dx.doi.org/10.33448/rsd-v10i5.14578

Petersen, P. E., Bourgeois, D., Ogawa, H., Estupinan-Day, S. \& Ndiaye, C. (2005). The global burden of oral diseases and risks to oral health. Bulletin of the World Health Organization, 83, 661-669. https://pubmed.ncbi.nlm.nih.gov/16211157/

Polit, D. F., Beck, C. T. \& Hungler, B. P. (2011). Fundamentos de pesquisa em enfermagem: métodos, avaliação e utilização. Artmed.

Rocha, B. S., Batista, H. A. S., Silva, A. M. S., Falcão, C. A. M., Bezerra, J. P., Batista, S. F., Ferraz, M. A. A. L. (2019). Patients with mental disorders: oral health condition. Focus Oral Research, 12(1), 30-34. http://www.focusoralresearch.com/index.php/for/article/view/39/pdf

Santos, D. L. N., Galvão-Moreira, L. V., Ribeiro, M. L., Santos, L. O., Cunha, J. R. T. \& Cruz, M. C. F. N. (2018). Multidisciplinary perspective on the oral health of individuals with mental disorders in a psychiatric institution. Rev de Pesquisa em Saúde, 19(1), 29-32. http://www.periodicoseletronicos.ufma.br/index.php/revistahuufma/article/viewFile/12170/6757

Sacchetto, M. S. L. S., Andrade, N. S., Brito, M. H. S. F., Lira, D. M. M. P., \& Barros, S. S. L. V. (2013). Evaluation of oral health in patients with mental disorders attended at the clinic of oral diagnosis of a public university. Revista de Odontologia da UNESP, 42(5), 344-349. https://dx.doi.org/10.1590/S180725772013000500005

Saraceno, B. (2011). A cidadania como forma de tolerância. Revista de Terapia Ocupacional da Universidade de São Paulo, 22(2):93-101. http://www.revistas.usp.br/rto/article/view/14126/15944

Seabra, P., Sarreira-Santos, A., Medeiros-Garcia, L., Costa, A., Cardoso, I. \& Figueiredo, A. S. (2018). A felicidade em pessoas com doença mental: uma revisão integrativa da literatura. Revista Portuguesa de Enfermagem de Saúde Mental, 19(45). http://dx.doi.org/10.19131/rpesm.0105

Sena, E. L. S., Ribeiro, B. S., Vanessa, S. T. C., Meira, V. S., Malhado, S. C. B. \& Carvalho, P. A. L. (2019). Percepção de familiares sobre a reabilitação psicossocial de alcoolistas. Revista Cubana de Enfermagem, 35(1). http://revenfermeria.sld.cu/index.php/enf/article/view/1851

U.S. Department of Health and Human Services (2016). U.S. Department of Health and Human Services Oral Health Strategic Framework, 2014-2017. Public health reports, $131(2), 242-257$. 